\title{
A Filter Model to Analyze Reconstruction Artifacts in Perfusion C-arm CT
}

\author{
Andreas Fieselmann, Frank Dennerlein, Yu Deuerling-Zheng, Jan Boese, \\ Rebecca Fahrig and Joachim Hornegger
}

\begin{abstract}
Interventional perfusion imaging with a C-arm angiography system capable of 3-D imaging (C-arm CT) could optimize the clinical workflow during stroke treatment. Acquisition times are currently of the order of several seconds per shortscan and filtered backprojection reconstruction artifacts can arise due to the time-dependence of the attenuation values. In this paper, we present a novel formalism to model reconstruction artifacts by a spatio-temporal filter in the object domain. The filter consists of terms that depend on the temporal derivatives of the attenuation values. Using this model, artifacts due to timedependent attenuation values can be well explained and studied. We apply the filter model to analyze redundancy weighting schemes in order to reduce these artifacts. Furthermore, this model could be the basis to develop new dynamic reconstruction algorithms.
\end{abstract}

\section{INTRODUCTION}

CT perfusion imaging can be used for stroke diagnosis. After injection of a contrast bolus a series of reconstructed images is obtained to track and analyze the contrast flow. Interventional perfusion imaging with a C-arm angiography system capable of 3-D imaging (Figure 1) could optimize the clinical workflow. Currently, the rotational speed of the C-arm is of the order of 3-5 seconds per short-scan. With the filtered backprojection (FBP) reconstruction artifacts can arise due to the time-dependence of the attenuation values.

To date there exist some studies on FBP artifacts due to time-varying attenuation values [1], [2], [3] but there is no model to describe the characteristics of these artifacts. Therefore, in this paper we present a novel formalism to model reconstruction artifacts by a spatio-temporal filter in the object domain with terms that depend on the temporal derivatives of the attenuation values and derivative-weighted point spread functions.

The filter model can be used to analyze reconstruction parameters (e.g. redundancy weighting schemes) in order to reduce the artifacts or to develop new dynamic reconstruction algorithms by inversion of this model.

\section{Preliminaries}

We assume that the X-ray source of the scanner rotates with a constant angular velocity $\omega_{\mathrm{s}}$ on a circular path of radius $R$

A.F. (e-mail: andreas.fieselmann@informatik.uni-erlangen.de) and J.H. are with the Pattern Recognition Lab and the Erlangen Graduate School in Advanced Optical Technologies (SAOT), University of Erlangen-Nuremberg, Erlangen, Germany. A.F., F.D., Y.D.-Z. and J.B. are with Siemens AG, Healthcare Sector, Forchheim, Germany. R.F. is with the School of Medicine, Stanford University, Stanford, CA, USA. The concepts and information presented in this paper are based on research and are not commercially available.

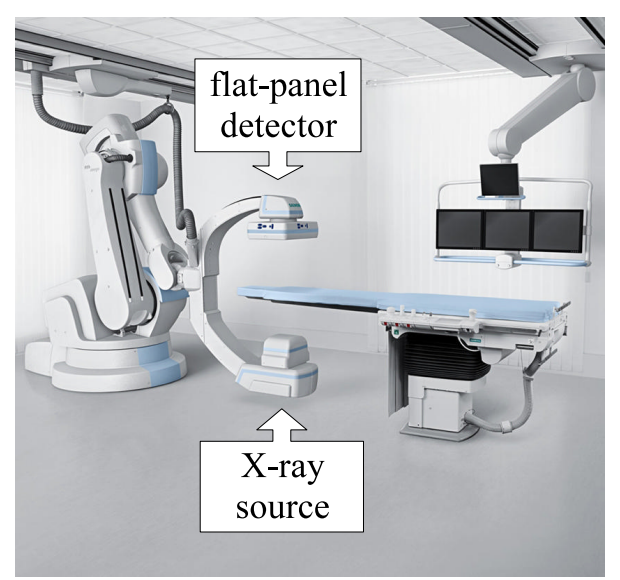

Figure 1. C-arm angiography system capable of CT-like imaging (C-arm CT; Artis zeego, Siemens AG).

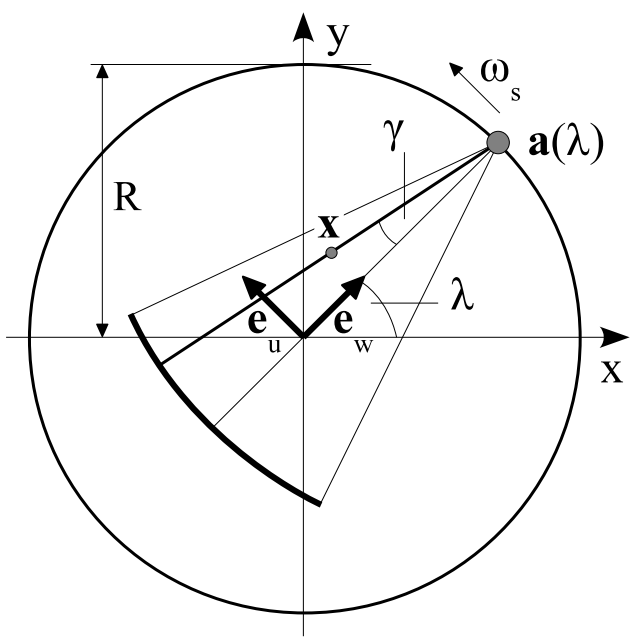

Figure 2. Fan-beam acquisition geometry described in Section II.

around the origin of the coordinate system (Figure 2). The location of the source $\mathbf{a}(\lambda(t))$ at time $t$ can be written as

$$
\begin{aligned}
\mathbf{a}(\lambda(t)) & =(R \cos (\lambda(t)), R \sin (\lambda(t)))^{\mathrm{T}} \\
\lambda(t) & =\omega_{\mathrm{s}} \cdot t+\lambda_{0}
\end{aligned}
$$

where $\lambda(t)$ is the angular position of the source at time $\mathbf{t}$ and $\lambda_{0}$ is the angle at $t=0$. For time-dependent attenuation values $\mu(\mathbf{x}, \lambda(t))$, an example is shown in Figure 3 , at locations $\mathbf{x}=(x, y)^{\mathrm{T}}$ the projections $p(\lambda(t), \gamma)$ under the fan angle $\gamma$ can be written with help of the usual definition of the delta 


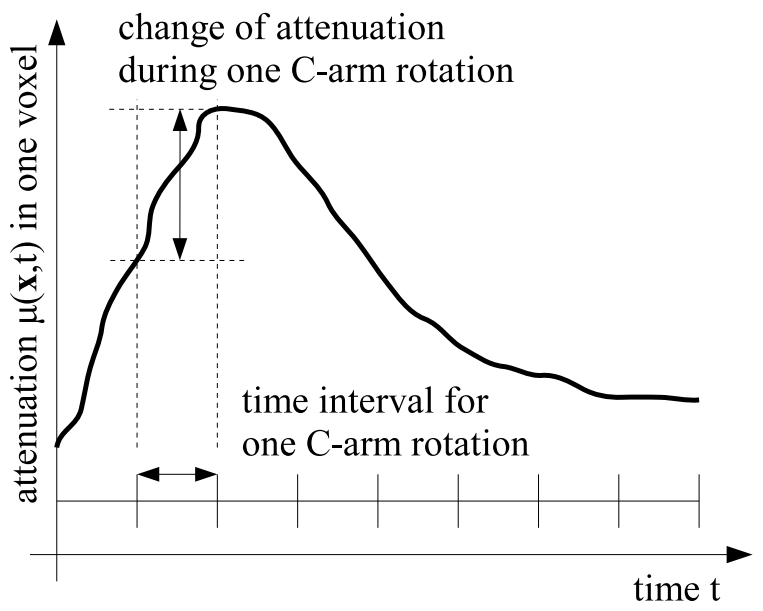

Figure 3. Example for a perfusion time-attenuation curve $\mu(\mathbf{x}, t)$ measured in one voxel at $\mathbf{x}$. The measured projections are inconsistent if the attenuation value changes during the $\mathrm{C}$-arm rotation.

function as

$$
p(\lambda(t), \gamma)=\iint_{-\infty}^{+\infty} \mu(\mathbf{x}, \lambda(t)) \cdot \delta\left(\gamma^{*}(\mathbf{x}, \lambda(t))-\gamma\right) \mathrm{d} x \mathrm{~d} y
$$

where $\gamma^{*}(\mathbf{x}, \lambda)$ defines the fan angle at source location $\lambda$ that intersects the pixel at location $\mathbf{x}$. This particular fan angle can be computed as

$$
\gamma^{*}(\mathbf{x}, \lambda)=\arctan \left(\frac{\mathbf{x} \cdot \mathbf{e}_{\mathrm{u}}(\lambda)}{R-\mathbf{x} \cdot \mathbf{e}_{\mathrm{w}}(\lambda)}\right)
$$

where we used the unit vectors $\mathbf{e}_{\mathrm{u}}=(-\sin (\lambda), \cos (\lambda))^{\mathrm{T}}$ and $\mathbf{e}_{\mathrm{w}}=(\cos (\lambda), \sin (\lambda))^{\mathrm{T}}$. We can reconstruct the object density $\mu_{\text {rec }}\left(\mathbf{r}, t_{\text {rec }}\right)$ at location $\mathbf{r}$ corresponding to the estimated object state at time $t_{\mathrm{rec}}$ by using the filtered backprojection [4]

$$
\begin{aligned}
& \mu_{\mathrm{rec}}\left(\mathbf{r}, t_{\mathrm{rec}}\right)=R \int_{-\infty}^{+\infty} \frac{1}{\|\mathbf{r}-\mathbf{a}(\lambda(t))\|^{2}} \int_{-\infty}^{+\infty} p(\lambda(t), \gamma) \\
& \cdot g\left(\gamma^{*}(\mathbf{r}, \lambda(t))-\gamma\right) \cdot \cos (\gamma) \cdot w\left(\lambda(t)-\lambda\left(t_{\mathrm{rec}}\right), \gamma\right) \mathrm{d} \gamma \mathrm{d} t
\end{aligned}
$$

with

$$
g(\gamma)=\left(\frac{\gamma}{\sin (\gamma)}\right)^{2} \cdot h_{\mathrm{ramp}}(\gamma)
$$

where $h_{\operatorname{ramp}}(\gamma)$ is the ramp filter [4]. The function $w(\lambda, \gamma)$ is a sliding window of length $\Lambda$. It is zero for $|\lambda| \geq \Lambda / 2$ and takes function values $m_{\Lambda}(\lambda, \gamma) \in[0,1]$ inside this angular interval to compensates for redundant data in the fan beam acquisition geometry. For $m_{\Lambda}(\lambda, \gamma)$ we choose the function proposed in [5].

Current C-arm CT systems cannot perform continuous rotations of the $\mathrm{C}$-arm but the $\mathrm{C}$-arm rotates in an alternating manner. Equation (5) therefore only applies to the data acquired during the rotation of the $\mathrm{C}$-arm in one direction. Furthermore, the geometry of the flat-panel detector is approximated by a curved detector.

\section{NOVEL FILTER Model}

Combining Equation (3) and Equation (5), changing the order of integration and evaluating the convolution with the delta function yields the equations

$$
\begin{gathered}
\mu_{\mathrm{rec}}\left(\mathbf{r}, t_{\mathrm{rec}}\right)=\iint_{-\infty}^{+\infty} \chi\left(\mathbf{x}, \mathbf{r}, t_{\mathrm{rec}}\right) \mathrm{d} x \mathrm{~d} y \\
\chi\left(\mathbf{x}, \mathbf{r}, t_{\mathrm{rec}}\right)=R \int_{-\infty}^{+\infty} \frac{1}{\|\mathbf{r}-\mathbf{a}(\lambda(t))\|^{2}} \cdot \mu(\mathbf{x}, \lambda(t)) \\
\cdot g\left(\gamma^{*}(\mathbf{r}, \lambda(t))-\gamma^{*}(\mathbf{x}, \lambda(t))\right) \cdot \cos \left(\gamma^{*}(\mathbf{x}, \lambda(t))\right) \\
\cdot w\left(\lambda(t)-\lambda\left(t_{\mathrm{rec}}\right), \gamma^{*}(\mathbf{x}, \lambda(t))\right) \mathrm{d} t
\end{gathered}
$$

We can interpret Equation (7) and Equation (8) as a filter that transforms the true attenuation values $\mu$ into the reconstructed attenuation values $\mu_{\mathrm{rec}}$. The function $\chi\left(\mathbf{x}, \mathbf{r}, t_{\mathrm{rec}}\right)$ can be interpreted as the contributions from locations $\mathbf{x}$ to the reconstruction at $\mathbf{r}$, i.e. it gives the reconstruction artifact due to time-varying attenuation values at $\mathbf{x}$.

We compute the Taylor expansion of $\mu(\mathbf{x}, \lambda(t))$ around $\lambda\left(t_{\mathrm{rec}}\right)$ in order to investigate the properties of $\chi$ with respect to derivatives of $\mu$.

$$
\mu(\mathbf{x}, \lambda(t))=\left.\sum_{n=0}^{\infty} \frac{\mathrm{d}^{n} \mu(\mathbf{x}, \lambda(t))}{\mathrm{d} \lambda^{n}}\right|_{t=t_{\mathrm{rec}}} \cdot \frac{\left(\lambda(t)-\lambda\left(t_{\mathrm{rec}}\right)\right)^{n}}{n !}
$$

Here we assume that $\mu(\mathbf{x}, \lambda(t))$ can be represented as a Taylor series in the time interval that corresponds to the current angular sliding window $w$. In practice this is reasonable since perfusion time attenuation curves are continuous, lowfrequency functions. According to Equation (2) second and higher-order derivatives of $\lambda(t)$ vanish, therefore we have the following total derivative

$$
\begin{aligned}
& \left.\frac{\mathrm{d}^{n} \mu(\mathbf{x}, \lambda(t))}{\mathrm{d} t^{n}}\right|_{t=t_{\mathrm{rec}}}= \\
& \left.\frac{\partial^{n} \mu(\mathbf{x}, \lambda(t))}{\partial \lambda^{n}}\right|_{t=t_{\mathrm{rec}}} \cdot\left(\left.\frac{\mathrm{d} \lambda(t)}{\mathrm{d} t}\right|_{t=t_{\mathrm{rec}}}\right)^{n}
\end{aligned}
$$

We combine Equation (8), Equation (9) and Equation (10) and change the order of summation and integration to obtain

$$
\chi\left(\mathbf{x}, \mathbf{r}, t_{\mathrm{rec}}\right)=\left.\sum_{n=0}^{\infty} \frac{\mathrm{d}^{n} \mu(\mathbf{x}, t)}{\mathrm{d} t^{n}}\right|_{t=t_{\mathrm{rec}}} \cdot \omega_{\mathrm{s}}^{-n} \cdot P_{n}\left(\mathbf{x}, \mathbf{r}, \lambda\left(t_{\mathrm{rec}}\right)\right)
$$

with

$$
\begin{aligned}
& P_{n}\left(\mathbf{x}, \mathbf{r}, \lambda\left(t_{\mathrm{rec}}\right)\right)=\frac{R}{n !} \int_{-\infty}^{+\infty} \frac{\left(\lambda(t)-\lambda\left(t_{\mathrm{rec}}\right)\right)^{n}}{\|\mathbf{r}-\mathbf{a}(\lambda(t))\|^{2}} \\
& \cdot g\left(\gamma^{*}(\mathbf{r}, \lambda(t))-\gamma^{*}(\mathbf{x}, \lambda(t))\right) \cdot \cos \left(\gamma^{*}(\mathbf{x}, \lambda(t))\right) \\
& \cdot w\left(\lambda(t)-\lambda\left(t_{\mathrm{rec}}\right), \gamma^{*}(\mathbf{x}, \lambda(t))\right) \mathrm{d} t
\end{aligned}
$$

We can interpret $P_{n}\left(\mathbf{x}, \mathbf{r}, \lambda\left(t_{\mathrm{rec}}\right)\right)$ as a derivative-weighted point spread function. It is weighted with the $n$-th derivative value of $\mu\left(\mathbf{x}, t_{\mathrm{rec}}\right)$ and the inverse X-ray source velocity $\omega_{\mathrm{s}}^{-n}$. The angle $\lambda\left(t_{\mathrm{rec}}\right)$ is the center of the sliding window. An illustration of the filter model is shown in Figure 4. For a 


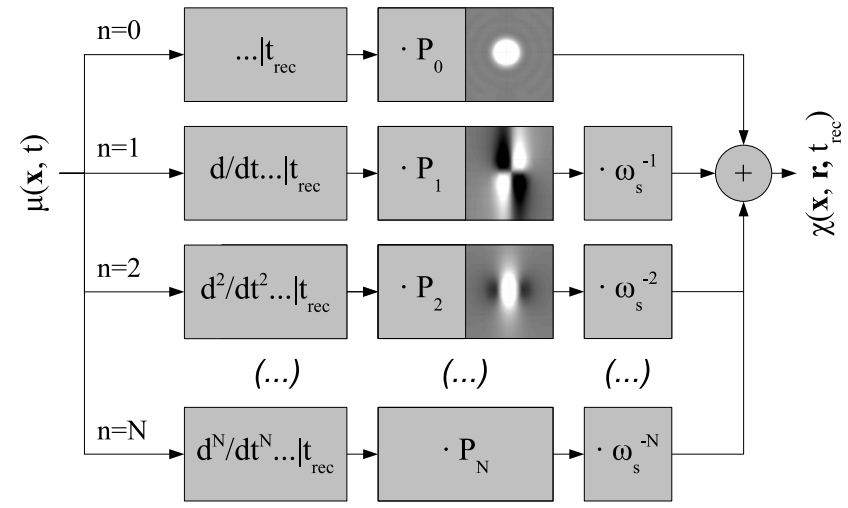

Figure 4. Illustration of the filter to model reconstruction of inconsistent projection data: The reconstruction artifact $\chi\left(\mathbf{x}, \mathbf{r}, t_{\mathrm{rec}}\right)$ around $\mathbf{x}$ arises from a varying attenuation value $\mu\left(\mathbf{x}, t_{\mathrm{rec}}\right)$ at $\mathbf{x}$. It is a superposition of derivativeweighted points spread functions $P_{n}$. For each term the weights consist of the $n$-th derivative of $\mu(\mathbf{x}, t)$ at $t_{\text {rec }}$ and a C-arm angular velocity $\left(\omega_{\mathrm{s}}\right)$ dependent factor.

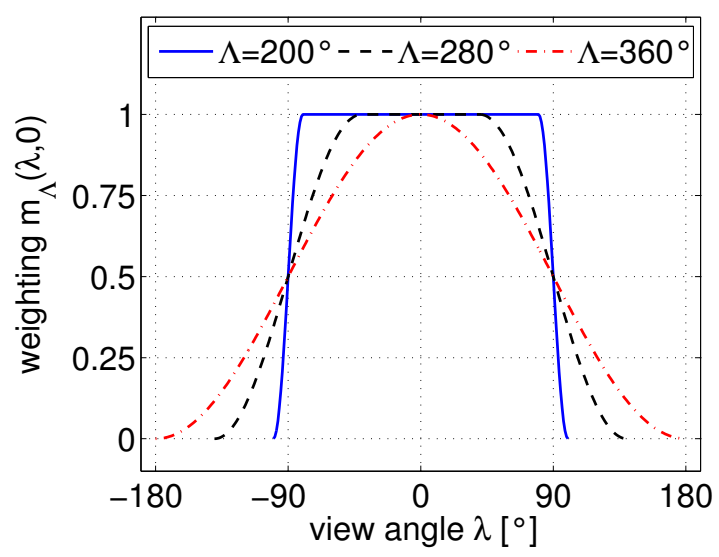

Figure 5. The redundancy weighting of the central ray according to the function proposed in [5] for different angular window intervals $\Lambda$.

reconstruction of a static object the filter model reduces to a convolution with the point spread function $P_{0}$. The function $P_{n}\left(\mathbf{x}, \mathbf{r}, \lambda\left(t_{\text {rec }}\right)\right)$ is shift-variant (it depends on $\left.\mathbf{r}\right)$ due to the fan-beam acquisition geometry but it is $2 \pi$ periodic with respect to $\lambda(t)$.

We have performed numerical simulations by forward and backprojections of different time-dependent attenuation value functions to confirm the mathematical derivation and the filter model showed excellent agreement with the reconstruction results.

\section{APPLICATION}

The filter model separates the temporal dynamics (derivatives of $\mu(\mathbf{x}, t), \mathrm{C}$-arm rotation speed $\left.\omega_{\mathrm{s}}\right)$ from the scan and reconstruction parameters that influence the point spread functions $P_{n}$. Therefore, it is possible to study the effect of different reconstruction parameters (e.g. $\Lambda, h_{\text {ramp }}$ ) on the artifacts by investigation of $P_{n}$.

Figure 5 shows examples of the redundancy weighting function $m_{\Lambda}(\lambda, 0)$ with different angular windows lengths $\Lambda$. Figure 6 shows plots of $P_{n}\left(\mathbf{x},(0,0)^{\mathrm{T}}, 0\right)$ computed for

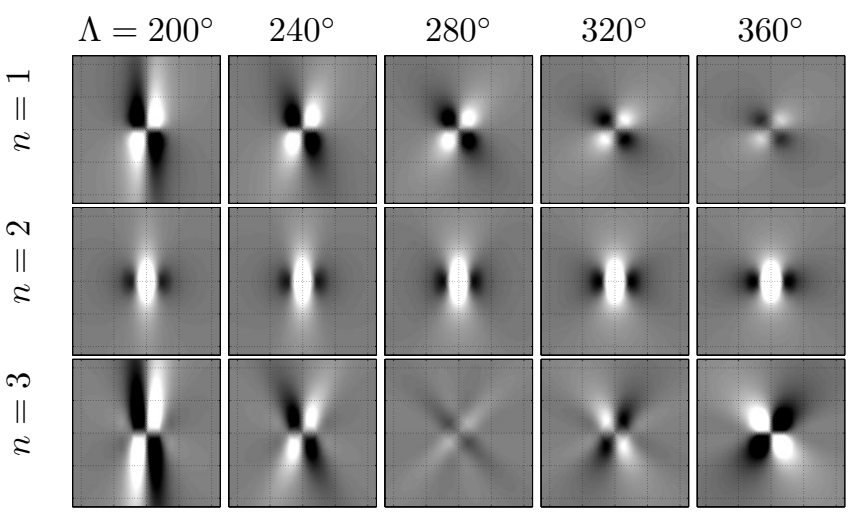

Figure 6. Derivative-weighted point spread functions $P_{n}$ of the artifact model computed for different sliding window length $\Lambda$. The window center is $0 \mathrm{HU}$ and the window widths are held constant for each $n$. The colormap range is from black to white.

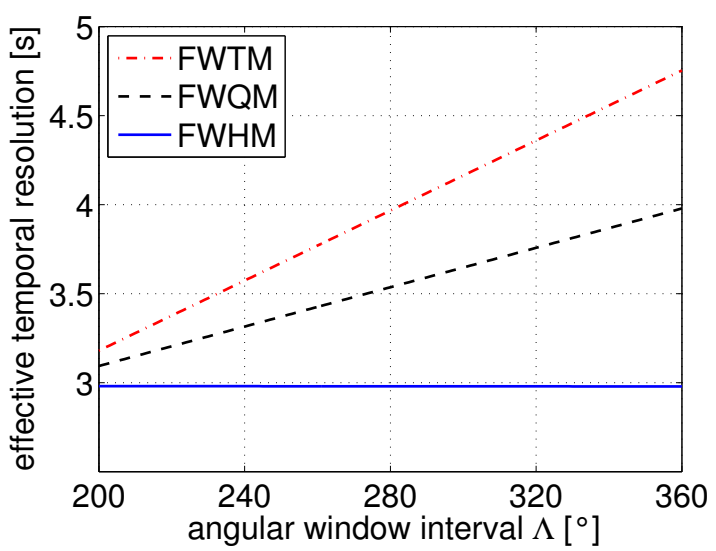

Figure 7. The effective temporal resolution given as the full width at half (FWHM), quarter (FWQM) and tenth (FWTM) maximum of the redundancy weighting function of the central ray for different angular window intervals $\Lambda$.

different $\Lambda$ and $n$. The window with $\Lambda=200^{\circ}$ represents the short-scan reconstruction. For this case the function $P_{1}$ has distinct streaks. They are reduced if $\Lambda=360^{\circ}$, i.e. if redundant data is included with a smooth weighting function. Artifacts that depend on higher-order $(n \geq 3)$ derivative values increase in magnitude for this $\Lambda$. However, since perfusion time attenuation curves are low frequency functions the contributions from lower-order derivatives are more important and we can optimize the sliding window length to suppress these contributions. Similar results were obtained empirically in [6] and can now be explained by this filter model from a different perspective.

The choice of $\Lambda$ influences the reconstruction artifact but also affects the temporal resolution of the reconstructed time attenuation curves. Figure 7 shows the effective temporal widths of the redundancy weighting function for different $\Lambda$ assuming a $\mathrm{C}$-arm rotation speed $w_{\mathrm{s}}=60^{\circ} / \mathrm{s}$. While the FWHM does not change the parameters FWQM and FWTM increase for increasing $\Lambda$. The temporal modulation transfer function could also be computed for different $\Lambda$ and could be the basis for further evaluations of the trade-off between the magnitude of the artifact and the temporal resolution.

Since all terms in the filter model (Equation (7) and (11)) 
are linear a numerical inversion of the model could be possible and lead to novel dynamic reconstruction algorithms.

\section{DiscusSiOn AND SUMmary}

We developed a novel formalism that can describe image reconstruction artifacts from inconsistent data due to timedependent attenuation values. It is well suited to describe artifacts in perfusion imaging. Typical time attenuation curves in perfusion imaging can be approximated as piecewise linear functions and thus only the components from a few derivative terms are sufficient to characterize the artifact.

The model yields a better understanding of the artifacts and can be applied to investigate their magnitude for given temporal dynamics. The influence of reconstruction parameters on the artifacts can be studied by investigation of the point spread functions of the model. Finally, new dynamic reconstruction algorithms could be developed based on this linear filter model, e.g. by deconvolution of the point spread functions.

The filter model can be easily extended to reconstruction algorithms that work with cone-beam data like the FDK algorithm [7]. In this case the equations in Section II must be adapted and the same principle in the derivation must be followed. The focus of our work is perfusion imaging with a C-arm CT but this filter model may also be of interest for other applications with slowly rotating scanners (e.g. dynamic SPECT or cone-beam CT in radiotherapy).

Interventional $\mathrm{C}$-arm $\mathrm{CT}$ perfusion measurement is a new application and a current area of research. It requires fast image reconstruction which can be achieved by using the FBP reconstruction. Our novel filter model is a mathematically exact analysis of the FBP algorithm in the presence of time-varying attenuation values. It contributes to a better understanding of these artifacts and it can improve the design of FBP-based reconstruction methods.

\section{ACKNOWLEDGMENT}

The authors gratefully acknowledge funding of the Erlangen Graduate School in Advanced Optical Technologies (SAOT) by the German Research Foundation (DFG) in the framework of the German excellence initiative.

\section{REFERENCES}

[1] J. E. Holden and W. R. Ip, "Continuous time-dependence in computed tomography," Medical Physics, vol. 5, no. 6, pp. 485-490, 1978.

[2] W. R. Ip, J. E. Holden, and S. S. Winkler, "A study of image discrepancies due to object time-dependence in transmission and emission tomography," Physics in Medicine and Biology, vol. 28, no. 8, pp. 953-962, 1983.

[3] B. D. Bok, A. N. Bice, M. Clausen, D. F. Wong, and H. N. Wagner, "Artifacts in camera based single photon emission tomography due to time activity variation," European Journal of Nuclear Medicine, vol. 13, no. 9, pp. 439-442, 1987.

[4] A. C. Kak and M. Slaney, Principles of Computerized Tomographic Imaging, IEEE Press, Philadelphia, PA, 1988.

[5] M. D. Silver, "A method for including redundant data in computed tomography," Medical Physics, vol. 27, no. 4, pp. 773-774, 2000.

[6] K. Taguchi, "Temporal resolution and the evaluation of candidate algorithms for four-dimensional CT," Medical Physics, vol. 30, no. 4, pp. 640-650, 2003.

[7] L. A. Feldkamp, L. C. Davis, and J. W. Kress, "Practical cone-beam algorithm," Journal of the Optical Society of America, vol. A1, pp. 612$619,1984$. 